\title{
Unipolar Depression
}

National Cancer Institute

\section{Source}

National Cancer Institute. Unipolar Depression. NCI Thesaurus. Code C35094.

A mood disorder having a clinical course involving one or more episodes of serious psychological depression that last two or more weeks each, do not have intervening episodes of mania or hypomania, and are characterized by a loss of interest or pleasure in almost all activities and by some or all of disturbances of appetite, sleep, or psychomotor functioning, a decrease in energy, difficulties in thinking or making decisions, loss of self-esteem or feelings of guilt, and suicidal thoughts or attempts. 